\title{
Répartition des paramètres physico-chimiques et métalliques des eaux usées de Hann (Dakar) avec le milieu marin
}

\author{
Ibrahima DIAGNE, Momar NDIAYE*, Birame NDIAYE, Cheikh Tidiane DIONE, \\ Dame CISSE, Maoudo HANE et Abdoulaye DIOP
}

Faculte des Sciences et Techniques, Laboratoire de Chimie Physique Organique et d'Analyse

Environnementale (LCPOAE)-UCAD, Dakar, Sénégal.

*Auteur correspondant, E-mail : momndiaye@hotmail.com

\section{RESUME}

Au Sénégal, les eaux usées constituent les principales sources de pollutions des milieux aquatiques. Ce travail étudie la répartition des paramètres physico-chimiques et métalliques des eaux usées drainées par le canal Est d'évacuation de la baie de Hann, en milieu marin. La commune de Hann, située le long du littoral dakarois, a été choisie pour les nombreuses activités, surtout industrielle qui s'y déroulent. L'Office Nationale de l'Assainissement du Sénégal (ONAS) estime à près de 180000 mètres cubes d'eaux usées par jour jetées en mer. Ainsi, l'environnement de la baie est agressé par les rejets industriels et domestiques sans traitement préalable. A cela, s'ajoute l'apport des effluents provenant du port de pêche, des petites industries de transformation, des habitats de type traditionnel et des décharges sauvages d'ordures. Les échantillons des mesures sont prélevés au point de chute des eaux du canal et à une distance de 40 mètres dans l'océan. Les paramètres physiques $(\mathrm{pH}$, température et conductivité) sont mesurés in situ à l'aide d'un appareil HANNA instruments $\mathrm{pH} /$ conductivity de marque HI 98129. Les paramètres chimiques (ammonium, chlorure, nitrate, phosphate, sulfate, fluorure) et métalliques (chrome 6, fer, potassium) ont été dosés par un spectrophotomètre de marque PF-11. Les résultats obtenus dans les eaux usées montrent la présence de tous les paramètres étudiés, notamment $\left(\mathrm{Cl}^{-}, \mathrm{PO}_{4}{ }^{3-}, \mathrm{NO}_{3}{ }^{-}, \mathrm{NH}_{4}{ }^{+}\right.$et $\left.\mathrm{SO}_{4}{ }^{2-}\right)$ de concentrations en moyennes respectives (223.33, $44.33,7.33,108.33$ et 161). Les teneurs notées en profondeur sont plus élevées que celles contenues dans l'eau de mer naturelle. Les valeurs maximales sont, cependant, observées près du point de rejet des eaux. Ainsi, ces eaux usées modifient la composition physico-chimique des eaux salines des côtes dakaroises jusqu'à $40 \mathrm{~m}$ du point de rejets.

(C) 2017 International Formulae Group. All rights reserved.

Mots clés: Eaux usées, eau de mer, paramètres physiques et métalliques, spectrophotométrie, interaction, contamination.

\section{Distribution of the physicochemical and metal parameters of worn water of Hann (Dakar) with the marine environment}

\begin{abstract}
In Senegal, worn water constitutes the principal sources of pollution of the aquatic environments. This work aimed at studying the distribution of the physicochemical and metal parameters of the worn water drained
\end{abstract}


by the channel East of evacuation of bay of Hann, in marine environment. The commune of Hann, located along the littoral of Dakar, was selected for many activities, especially industrial which proceed there. The National Office of the Cleansing of Senegal (ONAS) estimates at nearly 180000 cubic meters of water used per day thrown at sea. Thus, the environment of bay is attacked by the industrial and domestic wastes without preliminary treatment. With that, the contribution of the effluents coming from the fishing port is added, of the small processing industries, the habitats of the traditional type and the wild discharges of refuse. The samples of measurements are taken at the point of fall of water of the channel and at a distance of 40 meters in the ocean. The physical parameters $(\mathrm{pH}$, temperature and conductivity) are measured in situ using an apparatus HANNA instruments pH/conductivity of brand HI 98129. The chemical parameters (ammonium, chloride, nitrate, phosphate, sulphate, fluoride) and metal (chromium 6, iron, potassium) were proportioned by a spectrophotometer of mark PF-11. The results obtained in worn water show the presence of all the studied parameters, in particular $\left(\mathrm{Cl}^{-}, \mathrm{PO} 43^{-}, \mathrm{NO} 3{ }^{-}, \mathrm{NH} 4^{+}\right.$and $\left.\mathrm{SO} 42^{-}\right)$of concentrations in respective averages (223.33, 44.33, 7.33, 108.33 and 161). The noted contents in-depth are higher than those contained in natural sea water. The maximum values, however are observed close to the outlet of water. Thus, this worn water modifies the physicochemical composition of water saltworks of the coast of Dakar up to $40 \mathrm{~m}$ of the outlet.

(C) 2017 International Formulae Group. All rights reserved.

Keywords: Worn water, sea water, physicochemical, photometry, interaction, contamination.

\section{INTRODUCTION}

Les résidus industriels et domestiques déversés dans la mer ont des effets particulièrement nuisibles sur la vie marine. Plusieurs travaux ont montré que de nombreuses substances chimiques et industrielles entravent les réactions chimiques et provoquent la mort et parfois la disparition de certaines espèces (Cherigui et al., 2007). Cependant, l'augmentation de la température des eaux rejetées et de la dégradation des matières organiques provenant de celles-ci par les bactéries consomment l'oxygène du milieu aquatique, ce qui est néfaste pour la faune marine.

La qualité physico-chimique et métallique du milieu marin joue donc un rôle très important dans la détermination de la qualité biologique et du degré de la pollution de ce milieu. Les eaux usées déversées dans le milieu aquatique entrent aussi en interaction avec l'eau de mer. Dans ce milieu, tous les polluants, particulièrement les métaux lourds sont soumis au même titre que les substances naturelles, au jeu des phénomènes biogéochimiques (Maurice, 1996). Ces éléments peuvent se retrouver dans l'eau, à l'état de complexes minéraux ou organiques, sous forme colloïdales ou particulaires et se répartissent donc dans le milieu aquatique (Kunz et Jardim, 2000 ; Giokas et al., 2002). Les eaux usées urbaines constituent l'une des principales voies des métaux vers les écosystèmes aquatiques (Buzier et al., 2006). La transformation des éléments métalliques, due aux facteurs physico-chimiques ou biologiques, peut neutraliser ces métaux mais aussi, faciliter leur dispersion dans le milieu marin et augmenter ainsi leur degré de toxicité (Barkouch, 2007).

L'objectif de ce présent travail consistait à faire l'étude des paramètres physico-chimiques et métalliques des eaux usées du canal de la baie de Hann avant et après déversement au niveau de la mer pour évaluer l'influence du rejet dans ce milieu récepteur.

\section{MATERIEL ET METHODES}

\section{Sites de prélèvements et échantillonnage}

La région de Dakar reçoit une forte charge organique et minérale engendrée par les rejets domestiques et industriels. Dans le cadre de cette étude, la baie de Hann (Figure 1), situé le long de la côte dakaroise, a été choisie pour les nombreuses activités qui s'y déroulent. Le prélèvement des échantillons, dans des flacons en polyéthylène a été réalisé en trois campagnes (mars, avril et mai 2015) au niveau de différentes stations. Les échantillons ont été transportés au laboratoire et conservés entre 2 et $5{ }^{\circ} \mathrm{C}$ avant traitement et analyse (Berho, 2008). 


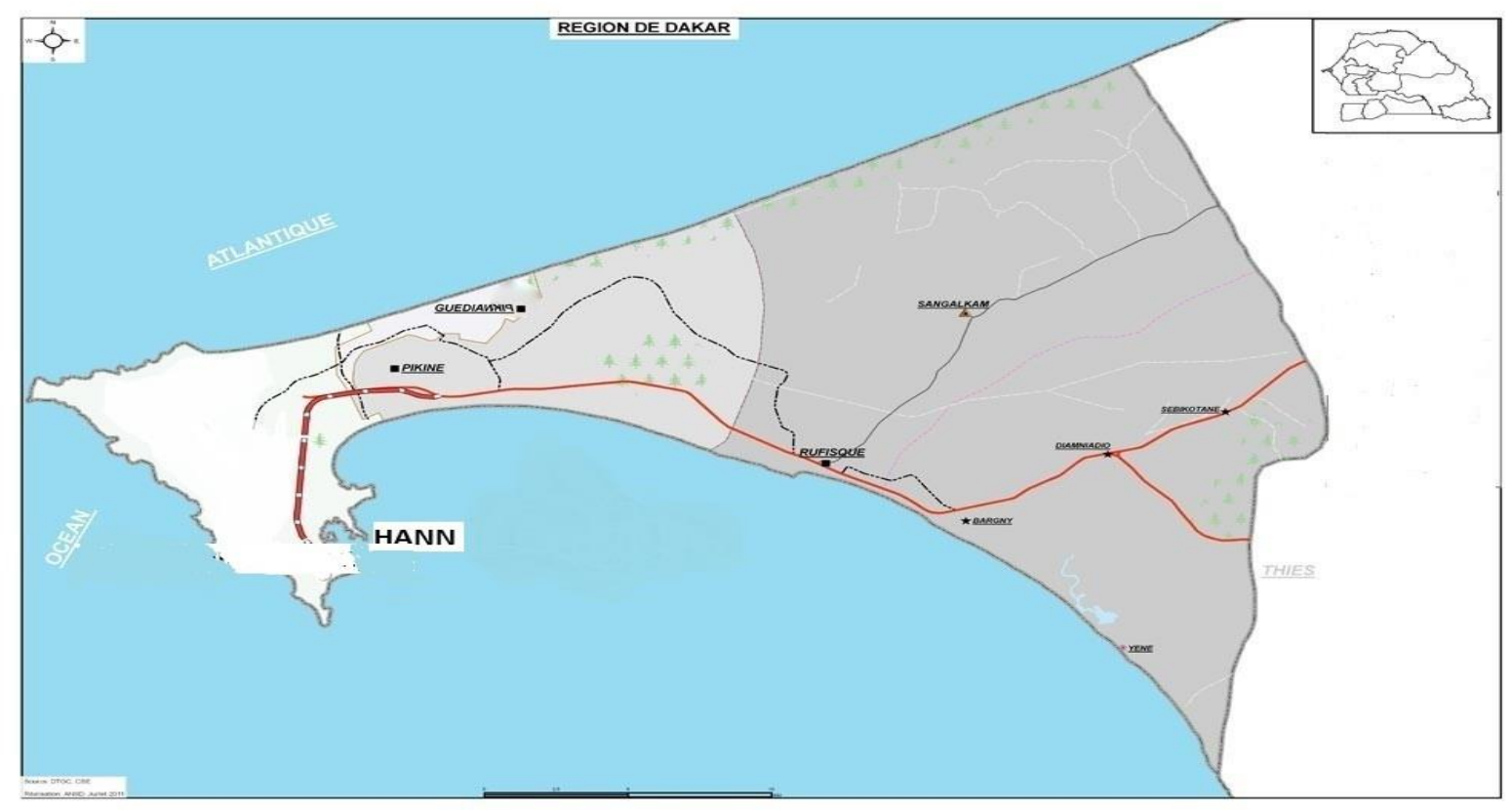

Figure 1 : Site de prélèvement des eaux.

\section{La commune de Hann}

La Baie de Hann est limitée à l'Est par le département de Dakar et le canal d'évacuation des eaux usées du port. Elle est la deuxième plus grande baie du monde après Rio. Mais aujourd'hui, une grande partie des entreprises industrielles du pays est installée au bord de cette baie, devenue le triste scénario d'un des plus grands dépotoirs d'ordures du Sénégal. En plus, une très grande diversité de polluants peut se retrouver dans l'eau, apportés par les eaux d'évacuation domestiques, industrielles et par les déversements accidentels ou illégaux de produits chimiques, de pétrole brut, d'huile usée, de déchets solides et de divers liquides.

\section{Mesure des paramètres physiques}

Les paramètres physiques (température, le $\mathrm{pH}$ et la conductivité) ont été mesurés in situ à l'aide d'un appareil HANNA instruments pH/conductivity HI 98129.

Pour la mesure du $\mathrm{pH}$, l'appareil a été étalonné avec des solutions tampons $\mathrm{pH}=7,01$ puis $\mathrm{pH}=4,01$.
Pour la conductivité, l'appareil a été calibré en immergeant la sonde dans la solution propre de calibrage HI 7031 (1413 $\mathrm{us} / \mathrm{cm})$.

La température s'affiche directement en bas et à gauche de l'écran lorsqu'on mesure le $\mathrm{pH}$ ou la conductivité.

\section{Les paramètres chimiques et métalliques}

Les paramètres chimiques et métalliques ont été mesurés par un photomètre PF-11 à cuve ronde.

\section{Préparation du blanc et de l'échantillon}

Le blanc peut être constitué de l'eau déminéralisée ou de l'échantillon à analyser sans ajout de réactifs.

La préparation de l'échantillon à analyser consiste à ajouter des réactifs dans 5 ou $10 \mathrm{~mL}$ d'échantillons d'eau prélevés. C'est une méthode d'analyse par ajout dosé. Il est très important de respecter le temps de lecture prescrit dans le protocole d'analyses pour assurer une homogénéité de la solution à doser. 


\section{Analyse des échantillons}

L'analyse des échantillons a été faite par la methode Visocolor, Visocolor Eco ou nanocolor. La méthode, à choisir, dépend de chaque élément et sa mesure s'affiche directement sur l'écran de l'appareil. D'abord, l'appareil a été calibré à $0 \mathrm{mg} / \mathrm{L}$ avec le blanc, avant chaque mesure. Ensuite, l'échantillon a été placé dans la cellule de mesure et le résultat de la mesure a été obtenu en $\mathrm{mg} / \mathrm{L}$ sur l'écran de l'appareil.

\section{RESULTATS ET DISCUSSION}

\section{Les eaux usées}

La caractérisation physico-chimique de ces eaux usées constitue un moyen pour estimer la qualité des rejets et leurs impacts sur le milieu récepteur. Le Tableau 1 récapitule les résultats des analyses des paramètres physiques (température, $\mathrm{pH}$, conductivité), chimiques (chlorures, fluorures, phosphates, sulfates, nitrates et ammonium) et métalliques (potassium, chrome 6 et fer) des échantillons d'eaux usées prélevées au niveau du canal d'évacuation de Hann.

\section{Interaction du rejet en mer}

Les résultats de cette étude montrent que les eaux usées peuvent avoir une influence non négligeable sur la composition physico-chimique et métallique de l'eau de mer.

\section{Paramètres physiques}

Les Figures 2 , 3 et 4 donnent respectivement l'évolution de la température, de la conductivité et du $\mathrm{pH}$ dans les différents points choisis de l'eau de mer de la baie de Hann.

La variation de la température ne dépasse pas une unité dans les différents points de prélèvements. Elle varie autour de $28,4{ }^{\circ} \mathrm{C}$. Cette faible variation peut être due à l'influence de la température de l'air mais pas de l'impact provenant du rejet. D'une manière générale, la température des eaux superficielles est influencée par la température de l'air et ceci d'autant plus que leur origine est moins profond (Rodier, 1997).
L'augmentation de la température s'accompagne d'une modification de la densité, d'une réduction de la viscosité, d'une augmentation de la tension de vapeur saturante à la surface et d'une diminution de la solubilité des gaz. Elle favorise aussi l'auto épuration et accroit la vitesse de sédimentation, ce qui peut présenter un intérêt dans les stations d'épuration (Djermakoye, 2005). Les élévations de température peuvent être nuisibles aux poissons, entraîner la mortalité de certaines espèces et favoriser le développement d'autres.

Le $\mathrm{pH}$ varie de 6.98 à 7.98 du point de rejet jusqu'à une distance de $40 \mathrm{~m}$. Cette différence est due à l'acidité entraînée par le rejet suivi d'une dilution de l'eau de mer. Les valeurs de $\mathrm{pH}$ mesurées dans les différents points sont très inférieures à celles de l'eau de mer naturelle. Il n'a pas de signification hygiénique mais il représente une notion importante de la détermination de l'agressivité de l'eau et la précipitation des éléments dissous (Abdesselem, 1999). Cependant, elles ne présentent pas de danger pour la faune et la flore, car seuls les pH inférieurs à 5 ou supérieurs à 9 sont mortelles pour les poissons (Diagne et al., 2017).

La conductivité décroît considérablement de 65085 à $60085 \mu \mathrm{S} / \mathrm{cm}$ du point de rejet jusqu'à l'intérieur de la mer. Ces valeurs obtenues dépassent largement la conductivité de l'eau mer naturelle. Elle permet d'apprécier rapidement mais très approximativement la minéralisation de l'eau et de suivre son évolution. Ainsi, on note une forte minéralisation des eaux de rejets qui ont influencé la conductivité du milieu récepteur. Ce paramètre permet aussi d'apprécier la quantité de sels dissous dans l'eau (Rodier et al., 1996).

\section{Paramètres chimiques}

Les Figures 5, 6, 7,8, 9 et 10 montrent que la concentration des chlorures est élevée au point de rejets $(25690 \mathrm{mg} / \mathrm{L})$ et diminue jusqu'à une distance de $40 \mathrm{~m}(22690 \mathrm{mg} / \mathrm{L})$. Ces concentrations dépassent la teneur des chlorures dans l'eau de mer naturelle. Ceci 
montre que, l'effet des eaux de rejet de la baie de Hann augmente la teneur du chlorure jusqu'à $40 \mathrm{~m}$ de profondeur du point de rejets. Les chlorures sont des ions importants contenus en concentrations variables dans les eaux naturelles, l'atmosphère, généralement sous forme de sels de sodium $(\mathrm{NaCl})$ et de potassium $(\mathrm{KCl})$. Ils représentent environ $0,05 \%$ de la lithosphère et sont souvent utilisés comme indice de pollution (Nechad et al., 2014). La concentration du fluorure est de $5.2 \mathrm{mg} / \mathrm{L}$ du point de rejet, et celle-ci diminue légèrement en fonction de la distance. Les concentrations trouvées dépassent largement la teneur en fluorure dans l'eau de mer naturelle. L'ion fluorure possède des propriétés basiques faibles, il forme de très puissantes liaisons hydrogène avec l'eau, ce qui explique sa stabilité en solution aqueuse. $\mathrm{La}$ toxicité des composés fluorés est proportionnelle à leur solubilité (Xavier, 2002). Cette toxicité des ions fluorures est de type chronique à savoir qu'elle se manifeste après des absorptions répétées de quantités relativement faibles. Il s'accumule dans les dents, les os et les reins mais il sera éliminé dans ces derniers dans les urines. A forte dose il peut endommager les dents (fluorose dentaire) et les os (fluorose osseuse) en diminuant le niveau de calcium (Houari et al., 2014).

Les teneurs en nitrates au niveau du point de rejet sont très faibles. Cette concentration augmente en fonction de la distance du point de rejets $(13 \mathrm{mg} / \mathrm{L})$ à $40 \mathrm{~m}$ de profondeur. Donc la teneur en nitrates dépasse largement la concentration naturelle de l'eau de mer. Par conséquent, on peut dire que ces fortes teneurs en nitrate dans le milieu récepteur peuvent avoir d'autres sources différentes des eaux de rejets. Ils sont présents dans le sol, dans les eaux superficielles et souterraines résultent de la décomposition naturelle, par des microorganismes, de matière organique azotée telle que les protéines végétales, animales et les excréments animaux. La principale action toxique du nitrate est due à la conversion de pigments porteurs d'oxygène à des formes incapables de transporter l'oxygène. Il existe une toxicité directe (pour les espèces qui y sont sensibles) et plusieurs biais de toxicité indirecte (liée à l'effet acidifiant du nitrate et à ses effets eutrophisants) qui conduisent notamment à la production d'algues.

Les concentrations en phosphates diminuent en fonction du point de rejets. Ce phénomène est dû à une dispersion des eaux usées brutes très riches en phosphate dans le milieu marin. Les eaux usées domestiques peuvent être chargées de détergents qui sont des produits contenant du phosphate. De même, l'urine rejetée dans l'environnement soit directement, soit par le biais des égouts contient aussi des phosphates. Le lessivage des terrains agricoles par les eaux de ruissellement est aussi une source de contamination $\mathrm{du}$ milieu récepteur. Les résultats montrent que jusqu'à $40 \mathrm{~m}$ du point de rejets, les concentrations en phosphates dépassent largement la teneur admise dans l'eau de mer. Les phosphates en eux-mêmes sont non toxiques pour la vie animale et végétale mais ils portent atteinte à l'environnement dès lors qu'ils sont en fortes concentrations. En effet, ils deviennent alors de véritables engrais pour les milieux aquatiques qu'ils contribuent à enrichir exagérément en matière organique perturbant ainsi les écosystèmes aquatiques. Ils constituent aussi l'une des causes majeures de l'eutrophisation (Nadjia, 2014).

Les teneurs en sulfates sont considérablement élevées du point de rejets allant jusqu'à $40 \mathrm{~m}$. Ces concentrations trouvées dans chacun des points sont très supérieures à la teneur admise dans l'eau de mer. Les sulfates peuvent être d'origine naturelle, biologique ou provenir de pollution industrielle et domestique. Selon l'OMS la teneur en sulfate dans l'eau de boisson ne doit pas dépasser $500 \mathrm{mg} / \mathrm{L}$. Pour des teneurs supérieures à ce seuil, l'eau peut être agressive et peut favoriser la corrosion des équipements de transport ou de distribution de l'eau donc peut entrainer des métaux lourds dans l'eau. 
La concentration en ammonium est très importante au niveau du point de déversement des eaux usées. Cependant, cette teneur diminue entre $10 \mathrm{~m}$ et $40 \mathrm{~m}$ de profondeur. Ainsi, la dispersion de l'ammonium provenant des eaux usées est presque totale à partir de 10 $\mathrm{m}$ du point de rejets. Sa présence dans les eaux traduit habituellement un processus de dégradation incomplète de la matière organique. Cette substance n'est pas réellement dangereuse pour la santé. Néanmoins, son inhalation peut provoquer des toux; son contact avec la peau ou les yeux des rougeurs peut entraîner des allergies; son ingestion, des nausées, des maux de gorge, des vomissements. Sur l'écosystème aquatique, il aboutit à l'eutrophisation (Portejoe et al., 2002).

\section{Paramètres métalliques}

Les résultats (Figures 11, 12 et 13) montrent que les concentrations en potassium sont inférieures à la valeur admise dans l'eau de mer, sauf au point de déversement et à 10 $\mathrm{m}$ de ce dernier. Ce métal s'oxyde rapidement au contact de l'air et réagit violemment avec l'eau. Il peut avoir des effets toxiques par inhalation. Ainsi, il peut entrainer une toux mais également l'irritation des yeux, du nez, de la gorge, des poumons avec un éternuement. Des expositions plus élevées peuvent causer une accumulation de liquide dans les poumons entrainant la mort. Le contact avec la peau et les yeux peut causer des brûlures graves menant à des dommages permanents.

La teneur en chrome (VI) est faible au point de rejets et presque nulle à partir de 10 $\mathrm{m}$ de ce dernier. Il s'accumule principalement dans les poumons avec une grande toxicité. Le Cr (VI) pénètre, particulièrement, les cellules à travers le système de transport des ions et il sera réduit en $\mathrm{Cr}$ (III), une fois à l'intérieur de la cellule (Goodale et al., 2008).

La concentration en fer est très remarquable au point de déversements des eaux provenant du canal d'évacuation des eaux usées. A partir de ce point, on enregistre une diminution des teneurs en fer en fonction de la distance. Mais malgré cette diminution au point de déversement et jusqu'à $40 \mathrm{~m}$ de profondeur, les teneurs de cet élément dépassent sa concentration dans l'eau de mer naturelle. Une surdose accidentelle de fer peut provoquer chez les sujets des dommages comme des lésions du foie, du cœur et des articulations. Elle peut aboutir, également, à l'hématochromatose (dépôt important de fer dans les tissus). Mais aussi on peut constater un œdème cérébral, une hypovolémie, une hypotension et parfois même un avortement spontané ou un accouchement prématuré (Hlavackova, 2005).

Tableau 1: les résultats des paramètres physico-chimiques et métalliques des eaux du canal.

\begin{tabular}{lcccc}
\hline Paramètres & Minimum & Maximum & Moyenne & Ecart type \\
\hline $\mathrm{pH}$ & 6.88 & 6.98 & 6.94 & 0.05 \\
$\mathrm{CE}(\mu \mathrm{S} / \mathrm{cm})$ & 1450 & 1735 & 1565 & 150.25 \\
$\mathrm{Cl}^{-}(\mathrm{ppm})$ & 180 & 250 & 223.33 & 37.86 \\
$\mathrm{~F}^{-}(\mathrm{ppm})$ & 0.9 & 1.2 & 1.06 & 0.15 \\
$\mathrm{PO}_{4}{ }^{3-}(\mathrm{ppm})$ & 40 & 48 & 44.33 & 4.04 \\
$\mathrm{NO}_{3}{ }^{-}(\mathrm{ppm})$ & 6 & 9 & 7.33 & 1.52 \\
$\mathrm{SO}_{4}{ }^{2-}(\mathrm{ppm})$ & 159 & 163 & 161 & 2.82 \\
$\mathrm{NH}_{4}{ }^{+}(\mathrm{ppm})$ & 100 & 115 & 108.33 & 7.33 \\
$\mathrm{~K}^{+}(\mathrm{ppm})$ & 19 & 20 & 19.66 & 0.57 \\
$\mathrm{Cr}^{6+}(\mathrm{ppm})$ & $<\mathrm{LD}$ & 0.02 & 0.01 & 0.01 \\
$\mathrm{Fe}(\mathrm{ppm})$ & 0.15 & 0.23 & 0.18 & 0.04 \\
\hline
\end{tabular}

LD : Limite de Détection. 


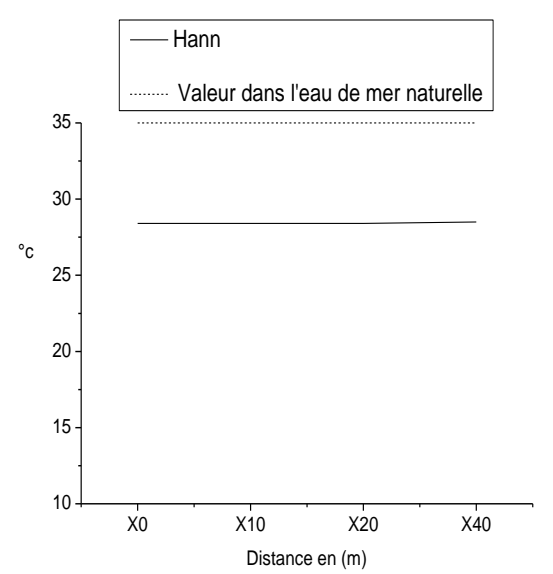

Figure 2: Variation de la température.

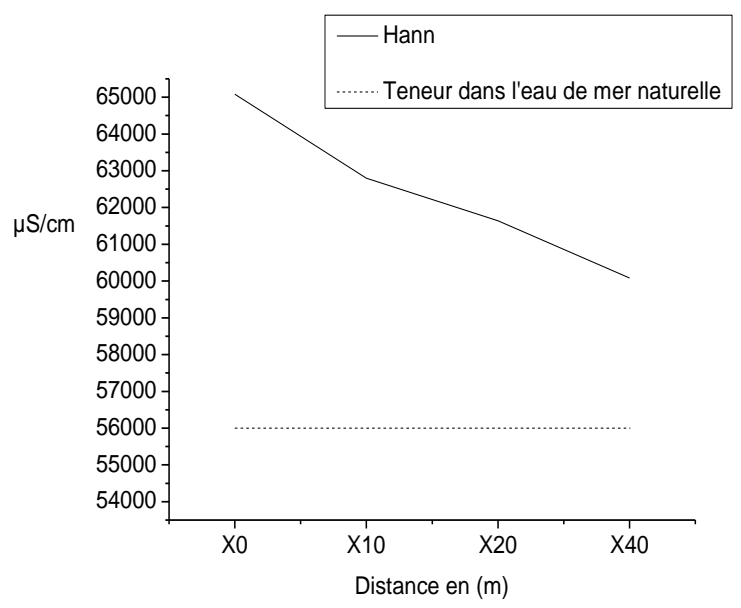

Figure 3: Variation de la conductivité.

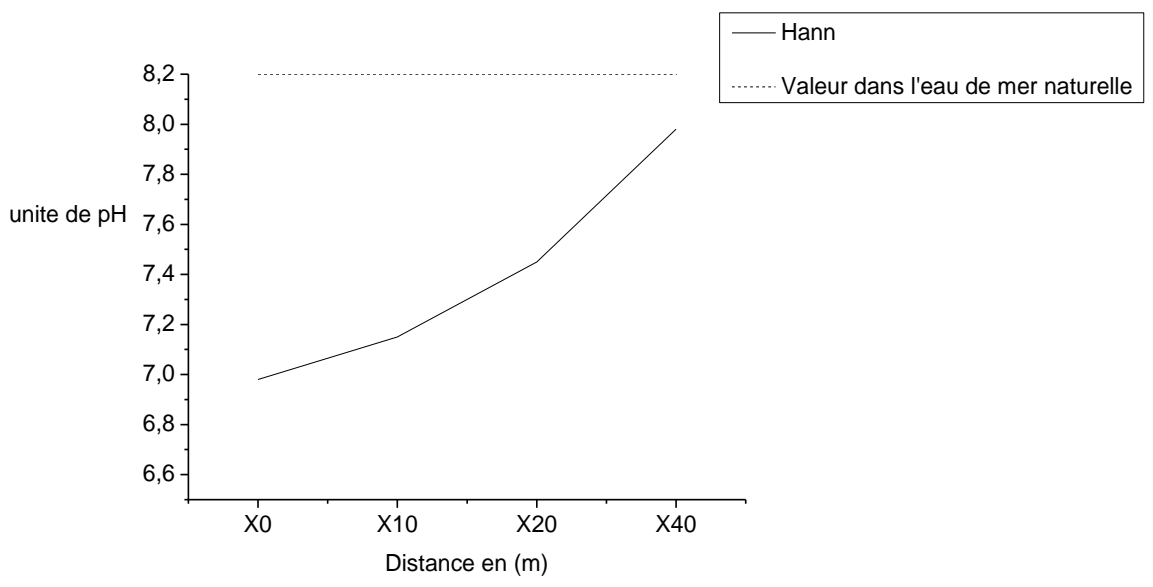

Figure 4 : Variation du $\mathrm{pH}$.

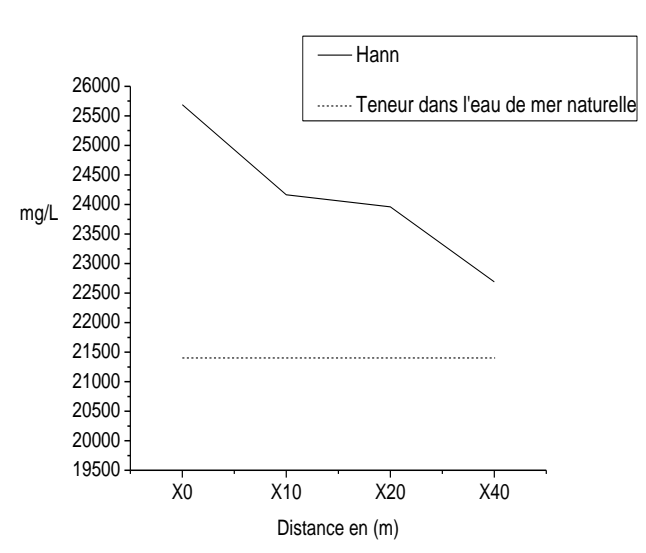

Figure 5 : Variation des chlorures.

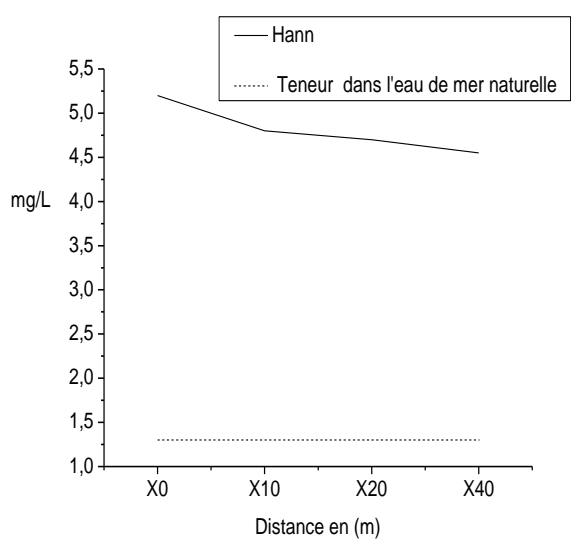

Figure 6 : Variation des fluorures. 


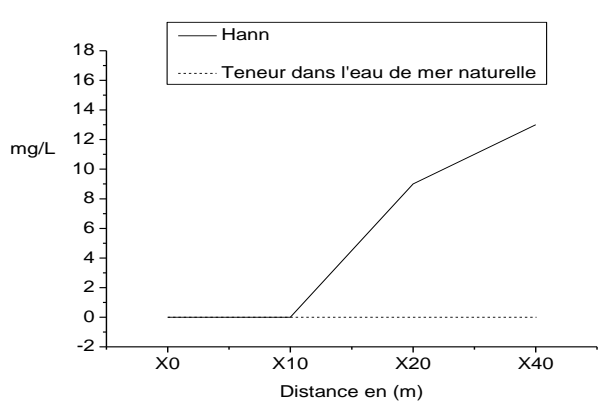

Figure 8 : Variation des phosphates.

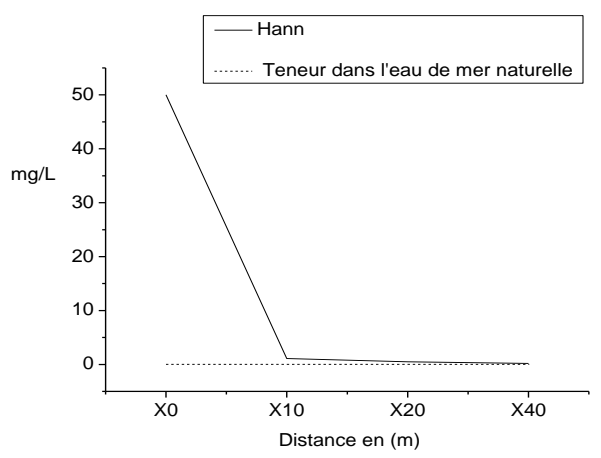

Figure 10 : Variation de l'ammonium.

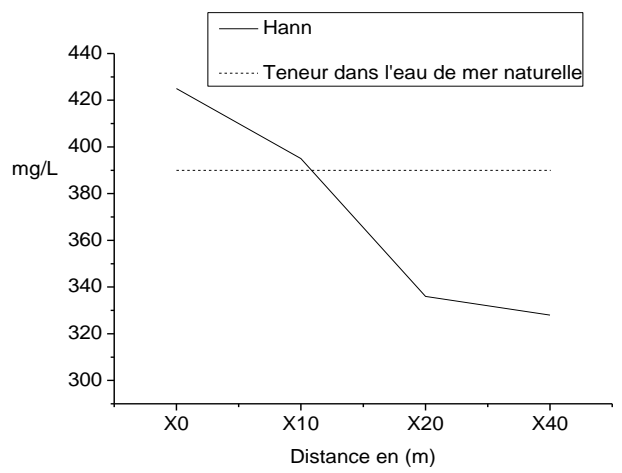

Figure 11 : Variation du potassium.

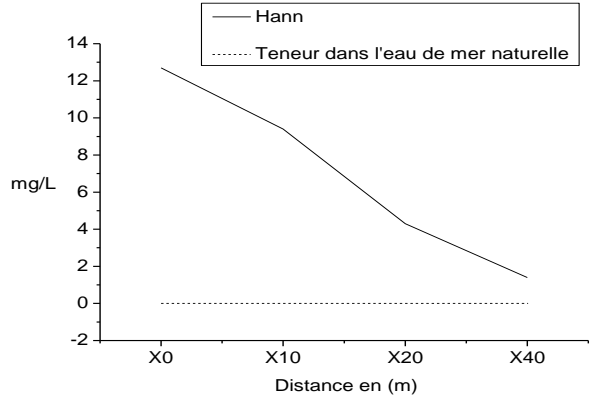

Figure 7 : Variation des nitrates.

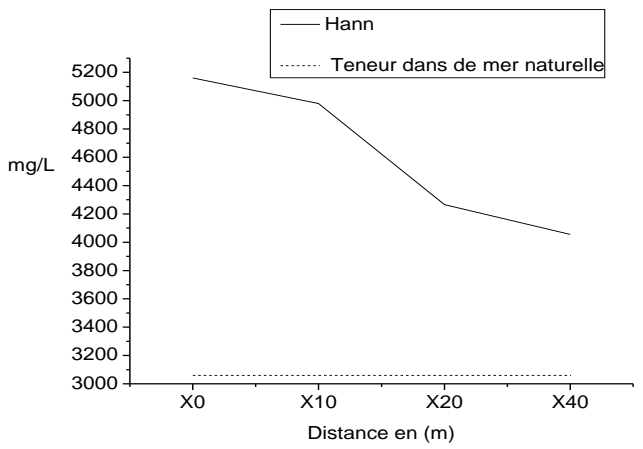

Figure 9 : Variation du sulfate.

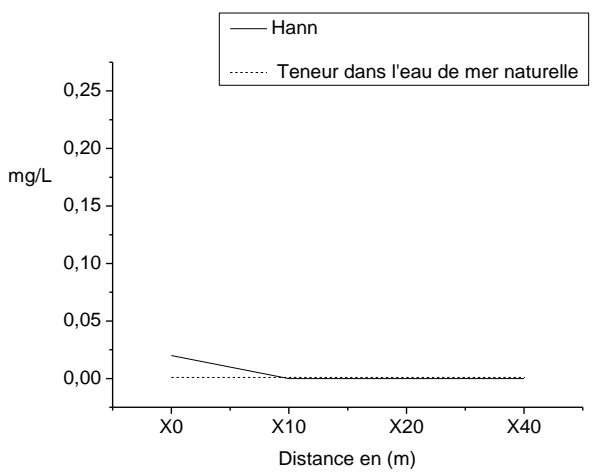

Figure 12 : Variation du chrome (6).

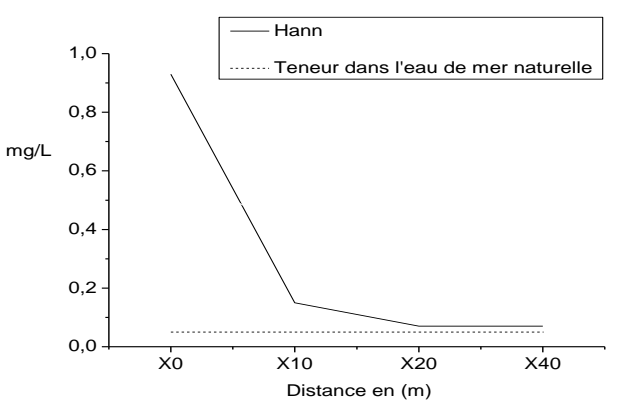

Figure 13 : Variation du fer. 


\section{Conclusion}

Les résultats, obtenus des paramètres physico-chimiques et métalliques au niveau des eaux de surface de la baie de Hann, montrent une dégradation de la qualité de l'eau, particulièrement au niveau des points de chute des eaux de rejets provenant du canal. Cependant, cette contamination a tendance à diminuer aux points situés loin du point de rejets. Cette diminution pourrait être liée au phénomène d'autoépuration. Ainsi, ces eaux usées contribuent, de manière importante, à la dégradation du milieu récepteur qui peut entrainer des problèmes de santé publique, par le phénomène de la bioamplification.

\section{CONTRIBUTIONS DES AUTEURS}

Les travaux ont été effectués au Laboratoire de Chimie Physique Organique et d'Analyse Environnementale (LCPOAE) du Departement de Chimie de l'Universite Cheikh Anta Diop de Dakar, dirigé par le Professeur Abdoulaye DIOP.

$$
\text { L'encadrement de l'équipe de }
$$
recherches est assuré par MN qui a élaboré le sujet et a supervisé le travail. ID est l'investigateur principal. CTD et DC ont participé à l'échantillonnage et à la préparation des solutions. MH a contribué à la réalisation des mesures. BN a participé à l'interprétation des résultats.

\section{REMERCIEMENTS}

Les auteurs remercient Macherey Nagel qui nous a offert le spectrophotomètre et les kits de standards et Monsieur Paul NKENG de l'Université de Strasbourg.

\section{REFERENCES}

Abdesselem A. 1999. Suivie de la Qualité Microbiologique et Physico-chimique de trois serres alimentant la région de Tlemcen. Mémoire d'ingénieur institut de biologie, université de Tlemcen. 218 p.
Barkouch Y. 2007. Etude du transfert des elements traces metalliques $(\mathrm{Al}, \mathrm{Cd}, \mathrm{Cu}$, $\mathrm{Pb}$, Se et $\mathrm{Zn}$ ) dans une chaine alimentaire d'une zone miniere de la region de Marrakech-Maroc. Thèse de doctorat de toxicologie. Université de NANTES, Faculté des Sciences Pharmaceutiques, $234 \mathrm{p}$.

Berho C. 2008. Procédures d'Echantillonnage des Eaux Souterraines en vue d'une analyse microbiologie. Etat de l'Art. Rapport final BRGM/RP-56405-FR, 26 $\mathrm{p}$.

Buzier R, Tusseau H, Mouchel J. 2006. Evaluation of DGT as a metal speciation tool in wastewater. Science of the Total Environment, 358: 277-285. DOI: 10.1016/j.scitotenv.2005.09.051

Cherigui HM, Palec G, Draoui A, Bournot P. 2007. Modélisation de la Propagation des Eaux Usées dans la Baie de Tanger. Albi : France; 5.

Diagne I, Drame S, Ndiaye B, Ndiaye M, Diop A. 2017. Caractérisation physicochimique et contamination métallique des eaux usées déversées au niveau de la Baie de Hann (Dakar/Sénégal). Int. J. Biol. Chem. Sci., 11(1): 462-472. DOI: http://dx.doi.org/10.4314/ijbcs.v11i1.37

Djermakoye M. 2005. Caractéristiques Physico-chimiques, Bactériologiques et Impact sur les Eaux de Surface et les Eaux Souterraines. Thèse de Doctorat, Université de Bamako, 135 p.

Giokas L, Antelo J, Paleologos E, Arce F, Karayannis M. 2002. Copper fractionation with dissolved organic matter in natural waters and wastewatera mixed micelle mediated methodology (cloud point extraction) employing flame atomic absorption spectrometry. $J$ Environ Monit., 4: 505-510. DOI: 10.1039/B202668F

Goodale BC, Walter R, Pelsue SR, Thompson WD, Wise SS, Winn RN, Mitani H, 
Wise JP. 2008. The cytotoxicity and genotoxicity of hexavalent chromium in medaka (Oryzias latipes). Aquat Toxicol., 1: 60-67. DOI : 10.1016/j.aquatox.2008.01.014

Hlavackova P. 2005. Evaluation du comportement du cuivre et du zinc dans une matrice de type sol à l'aide de différentes méthodologiques, thèse, Institut National des Sciences de Lyon, Ecole doctorale : chimie de Lyon, $202 \mathrm{p}$. Houari S, Wurtz T, Ferbus D, Chateau D, Dessombz A, Berdal A, Babajko S. 2014. Asporin and the mineralization process in fluoride-treated rats. $J$ Bone Miner, 29: 1446-1455. DOI: $10.1002 / \mathrm{jbmr} .2153$

Kunz A, Jardim WF. 2000. Complexation and adsorption of copper in raw sewage. Water Res., 34 : 2061-2068.

Maurice M. 1996. L'Ecotoxicologie Appliquée du Milieu Marin. R.int.Del/NANTES ; 104 p.

Nadjia B. 2014. Elimination du phosphore sous la forme des phosphates Par adsorption sur la diatomite brute et diatomite modifiée au fer- FHMD. Mémoire, contrôle et gestion de l'environnement. Université des Sciences et de la Technologie d'ORAN. 86 p.

Nechad I, Fadil k, Fadil F. 2014. Qualité Physicochimiques des Eaux des sources Ain Regrag et Ain Bouali dans la région de Séfrou (moyen atlas, Maroc). Larhyss Journal, 20: 127-146.

Portejoie S, Martinez J, Landmann G. 2002. L'ammoniac d'Origine Agricole: impacts sur la santé humaine et animale et sur les milieux naturels, 151-160.

Rodier J. 1997. L'Analyse de l'Eau (Eaux Naturelles, Eaux Résiduaires et Eaux de Mer) ( $8^{\text {ème }}$ edn). Dunod : Paris ; 66.

Rodier J, Legube B, Merlet N. 1996. L'Analyse de l'Eau, (8è édn). Dunod: Paris ; 1384.

Xavier ML. 2002. Contribution à l'étude de l'évolution de la fluorose en Maurienne. Thèse d'état, Ecole nationale vétérinaire de Lyon, p 258. 